\title{
StNet: Local and Global Spatial-Temporal Modeling for Action Recognition
}

\author{
Dongliang He, ${ }^{1}$ Zhichao Zhou, ${ }^{1}$ Chuang Gan, ${ }^{2} \mathbf{F u ~} \mathrm{Li}^{1}$ \\ Xiao Liu, ${ }^{1}$ Yandong Li, ${ }^{3}$ Limin Wang, ${ }^{4}$ Shilei Wen ${ }^{1}$ \\ Department of Computer Vision Technology (VIS), Baidu Inc. ${ }^{1}$ \\ MIT-IBM Watson AI Lab ${ }^{2}$, University of Central Florida ${ }^{3}$ \\ State Key Lab for Novel Software Technology, Nanjing University, China ${ }^{4}$ \\ \{hedongliang01, zhouzhichao01, lifu, liuxiao12,wenshilei\}@baidu.com \\ ganchuang1990@gmail.com, lyndon.leeseu@outlook.com, lmwang.nju@gmail.com
}

\begin{abstract}
Despite the success of deep learning for static image understanding, it remains unclear what are the most effective network architectures for spatial-temporal modeling in videos. In this paper, in contrast to the existing $\mathrm{CNN}+\mathrm{RNN}$ or pure 3D convolution based approaches, we explore a novel spatialtemporal network (StNet) architecture for both local and global modeling in videos. Particularly, StNet stacks $N$ successive video frames into a super-image which has $3 N$ channels and applies 2D convolution on super-images to capture local spatial-temporal relationship. To model global spatialtemporal structure, we apply temporal convolution on the local spatial-temporal feature maps. Specifically, a novel temporal Xception block is proposed in StNet, which employs a separate channel-wise and temporal-wise convolution over the feature sequence of a video. Extensive experiments on the Kinetics dataset demonstrate that our framework outperforms several state-of-the-art approaches in action recognition and can strike a satisfying trade-off between recognition accuracy and model complexity. We further demonstrate the generalization performance of the leaned video representations on the UCF101 dataset.
\end{abstract}

\section{Introduction}

Action recognition in videos has received significant research attention in the computer vision and machine learning community (Karpathy et al. 2014; Wang and Schmid 2013; Wang, Qiao, and Tang 2016; Simonyan and Zisserman 2014; Fernando et al. 2015; Wang et al. 2016; Qiu, Yao, and Mei 2017; Carreira and Zisserman 2017; Shi et al. 2017; Zhang et al. 2016). The increasing ubiquity of recording devices has created videos far surpassing what we can manually handle. It is therefore desirable to develop automatic video understanding algorithms for various applications, such as video recommendation, human behavior analysis, video surveillance and so on. Both local and global information is important for this task, as shown in Fig.1. For example, to recognize "Laying Bricks" and "Laying Stones", local spatial information is critical to distinguish bricks and stones; and to classify "Cards Stacking" and "Cards Flying", global spatial-temporal clues are the key evidence.

Copyright (C) 2019, Association for the Advancement of Artificial Intelligence (www.aaai.org). All rights reserved.

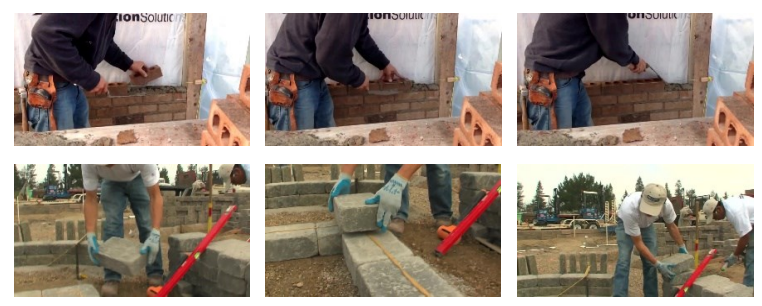

(a) "Laying Bricks" (Top) v.s. "Laying Stone" (Bottom)
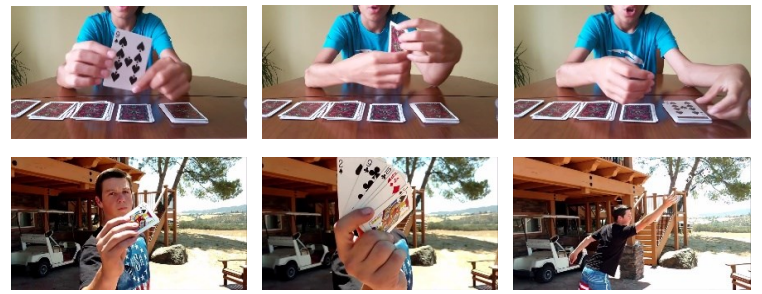

(b) "Cards Stacking” (Top) v.s. "Cards Flying” (Bottom)

Figure 1: Local information is sufficient to distinguish "Laying Bricks" and "Laying Stones" while global spatialtemporal clue is necessary to tell "Cards Stacking" and "Cards Flying".

Motivated by the promising results of deep networks (Ioffe and Szegedy 2015; He et al. 2016; Szegedy et al. 2017) on image understanding tasks, deep learning is applied to the problem of video understanding. Two major research directions are explored specifically for action recognition, i.e., employing $\mathrm{CNN}+\mathrm{RNN}$ architectures for video sequence modeling (Donahue et al. 2015; Yue-Hei Ng et al. 2015) and purely deploying ConvNet-based architectures for video recognition (Simonyan and Zisserman 2014; Feichtenhofer, Pinz, and Wildes 2016; 2017; Wang et al. 2016; Tran et al. 2015; Carreira and Zisserman 2017; Qiu, Yao, and Mei 2017).

Although considerable progress has been made, current action recognition approaches still fall behind human performance in terms of action recognition accuracy. The main challenge lies in extracting discriminative spatial-temporal representations from videos. For the CNN+RNN solution, 
the feed-forward CNN part is used for spatial modeling, while the temporal modeling part, i.e., LSTM (Hochreiter and Schmidhuber 1997) or GRU (Cho et al. 2014), makes end-to-end optimization very difficult due to its recurrent architecture. Nevertheless, separately training CNN and RNN parts is not optimal for integrated spatial-temporal representation learning.

ConvNets for action recognition can be generally categorized into 2D ConvNet and 3D ConvNet. 2D convolution architectures (Simonyan and Zisserman 2014; Wang et al. 2016) extract appearance features from sampled RGB frames, which only exploit local spatial information rather than local spatial-temporal information. As for the temporal dynamics, they simply fuse the classification scores obtained from several snippets. Although averaging classification scores of several snippets is straightforward and efficient, it is probably less effective for capturing spatiotemporal information. C3D (Tran et al. 2015) and I3D (Carreira and Zisserman 2017) are typical 3D convolution based methods which simultaneously model spatial and temporal structure and achieve satisfying recognition performance. As we know, compared to deeper network, shallow network exhibits inferior capacity on learning representation from large scale datasets. When it comes to large scale human action recognition, on one hand, inflating shallow 2D ConvNets to their 3D counterparts may be not capable enough of generating discriminative video descriptors; on the other hand, 3D versions of deep 2D ConvNets will result in too big model as well as too heavy computation cost both in training and inference phases.

Given the aforementioned concerns, we propose our novel spatial-temporal network (StNet) to tackle the large scale action recognition problem. First, we consider local spatialtemporal relationship by applying $2 \mathrm{D}$ convolution on $3 \mathrm{~N}$ channel super-image, which is composed of $\mathrm{N}$ successive video frames. Thus local spatial-temporal information can be more efficiently encoded compared to 3D convolution on $\mathrm{N}$ images. Second, StNet inserts temporal convolutions upon feature maps of super-images to capture temporal relationship among them. Local spatial-temporal modeling followed by temporal convolution can progressively builds global spatial-temporal relationship and is lightweight and computational friendly. Third, in StNet, the temporal dynamics are further encoded with our proposed temporal Xception block (TXB) instead of averaging scores of several snippets. Inspired by separable depth-wise convolution (Chollet 2017), TXB encodes temporal dynamics in a separate channel-wise and temporal-wise 1D convolution manner for smaller model size and higher computation efficiency. Finally, TXB is convolution based rather than recurrent architecture, it is easily to be optimized via stochastic gradient decent (SGD) in an end-to-end manner.

We evaluate the proposed StNet framework over the newly released large scale action recognition dataset Kinetics (Kay et al. 2017). Experiment results show that StNet outperforms several state-of-the-art 2D and 3D convolution based solutions, meanwhile our StNet attains better efficiency from the perspective of the number of FLOPs and higher effectiveness in terms of recognition accuracy than its 3D CNN counterparts. Besides, the learned representation of StNet is transferred to the UCF101 (Soomro, Zamir, and Shah 2012) dataset to verify its generalization capability.

\section{Related Work}

In the literature, video-based action recognition solutions can be divided into two categories: action recognition with hand-crafted features and action recognition with deep ConvNet. To develop effective spatial-temporal representations, researchers have proposed many hand-crafted features such as HOG3D (Klaser, Marszałek, and Schmid 2008), SIFT3D (Scovanner, Ali, and Shah 2007), MBH (Dalal, Triggs, and Schmid 2006). Currently, improved dense trajectory (Wang and Schmid 2013) is the state-of-the-art among the handcrafted features. Despite its good performance, such handcrafted feature is designed for local spatial-temporal description and is hard to capture semantic level concepts. Thanks to the big progress made by introducing deep convolution neural network, ConvNet based action recognition methods have achieved superior accuracy to conventional handcrafted methods. As for utilizing $\mathrm{CNN}$ for video-based action recognition, there exist the following two research directions:

Encoding CNN Features: CNN is usually used to extract spatial features from video frames, and the extracted feature sequence is then modelled with recurrent neural networks or feature encoding methods. In LRCN (Donahue et al. 2015), CNN features of video frames are fed into LSTM network for action classification. ShuttleNet (Shi et al. 2017) introduced biologically-inspired feedback connections to model long-term dependencies of spatial CNN descriptors. TLE (Diba, Sharma, and Van Gool 2017) proposed temporal linear encoding that captures the interactions between video segments, and encoded the interactions into a compact representations. Similarly, VLAD (Arandjelovic et al. 2016; Girdhar et al. 2017) and AttentionClusters (Long et al. 2018) have been proposed for local feature integration.

ConvNet as Recognizer: the first attempt to use deep convolution network for action recognition was made by Karpathy et.al, (Karpathy et al. 2014). While strong results for action recognition have been achieved by (Karpathy et al. 2014), two stream ConvNet (Simonyan and Zisserman 2014) that merges the predicted scores from a RGB based spatial stream and an optical flow based temporal stream obtained performance improvements in a large margin. STResNet (Feichtenhofer, Pinz, and Wildes 2016) introduced residual connections between the two streams of (Simonyan and Zisserman 2014) and showed great advantage in results. To model the long-range temporal structure of videos, Temporal Segment Network (TSN) (Wang et al. 2016) was proposed to enable efficient video-level supervision by sparse temporal sampling strategy and further boosted the performance of ConvNet based action recognizer.

Observing that 2D ConvNets cannot directly exploit the temporal patterns of actions, spatial-temporal modeling techniques were involved in a more explicit way. C3D (Tran et al. 2015) applied 3D convolutional filters to the videos 


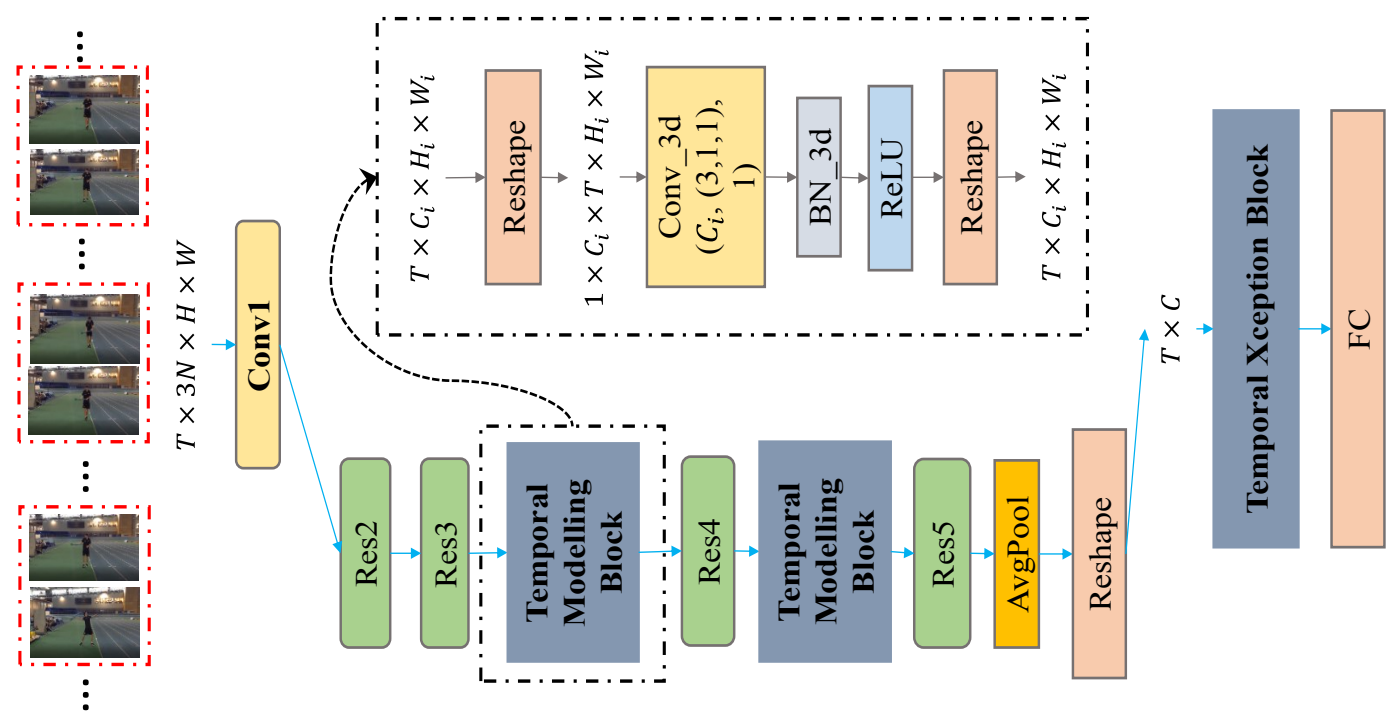

Figure 2: Illustration of constructing StNet based on ResNet (He et al. 2016) backbone. The input to StNet is a $T \times 3 N \times H \times W$ tensor. Local spatial-temporal patterns are modelled via 2D Convolution. 3D convolutions are inserted right after the Res3 and Res4 blocks for long term temporal dynamics modelling. The setting of 3D convolution (\# Output Channel, (temporal kernel size, height kernel size, width kernel size), \# groups) is $\left(C_{i},(3,1,1), 1\right)$.

to learn spatial-temporal features. Compared to 2D ConvNets, C3D has more parameters and is much more difficult to obtain good convergence. To overcome this difficulty, T-ResNet (Feichtenhofer, Pinz, and Wildes 2017) injects temporal shortcut connections between the layers of spatial ConvNets to get rid of 3D convolution. I3D (Carreira and Zisserman 2017) simultaneously learns spatial-temporal representation from video by inflating conventional 2D ConvNet architecture into 3D ConvNet. P3D (Qiu, Yao, and Mei 2017) decouples a 3D convolution filter to a 2D spatial convolution filter followed by a $1 \mathrm{D}$ temporal convolution filter. Recently, there are many frameworks proposed to improve 3D convolution (Zolfaghari, Singh, and Brox 2018; Wang et al. 2018a; Xie et al. 2018; Tran et al. 2018; Wang et al. 2018b; Chen et al. 2018). Our work is different in that spatial-temporal relationship is progressively modeled via temporal convolution upon local spatial-temporal feature maps.

\section{Proposed Approach}

The proposed StNet can be constructed from the existing state-of-the-art 2D ConvNet frameworks, such as ResNet (He et al. 2016), InceptionResnet (Szegedy et al. 2017) and so on. Taking ResNet as an example, Fig.2 illustrates how we can build StNet from the existing 2D ConvNet. It is similar to build StNet from other 2D ConvNet frameworks such as InceptionResnetV2 (Szegedy et al. 2017), ResNeXt (Xie et al. 2017) and SENet (Hu, Shen, and Sun 2018). Therefore, we do not elaborate all such details here.

Super-Image: Inspired by TSN (Wang et al. 2016), we choose to model long range temporal dynamics by sampling temporal snippets rather than inputting the whole video se- quence. One of the differences from TSN is that we sample $T$ temporal segments each of which consists of $N$ consecutive RGB frames rather than a single frame. These $N$ frames are stacked in the channel dimension to form a $s u$ per image, so the input to the network is a tensor of size $T \times 3 N \times H \times W$. Super-Image contains not only local spatial appearance information represented by individual frame but also local temporal dependency among these successive video frames. In order to jointly modeling the local spatialtemporal relationship therein and as well as to save model weights and computation costs, we leverage $2 \mathrm{D}$ convolution (whose input channel size is $3 N$ ) on each of the $T$ superimages. Specifically, the local spatial-temporal correlation is modeled by 2D convolutional kernels inside the Conv1, Res2, and Res3 blocks of ResNet as shown in Fig.2. In our current setting, $N$ is set to 5 . In the training phase, $2 \mathrm{D}$ convolution blocks can be initialized directly with weights from the ImageNet pre-trained backbone 2D convolution model except the first convolution layer. Weights of Conv1 can be initialized following what the authors have done in I3D (Carreira and Zisserman 2017).

Temporal Modeling Block: 2D convolution on the $T$ super-images generates $T$ local spatial-temporal feature maps. Building the global spatial-temporal representation of the sampled $T$ super-images is essential for understanding the whole video. Specifically, we choose to insert two temporal modeling blocks right after the Res3 and Res4 block. The temporal modeling blocks are designed to capture the long-range temporal dynamics inside a video sequence and they can be easily implemented by leveraging the architecture of Conv3d-BN3d-ReLU. Note that the existing 2D ConvNet framework is powerful enough for spatial modeling, 


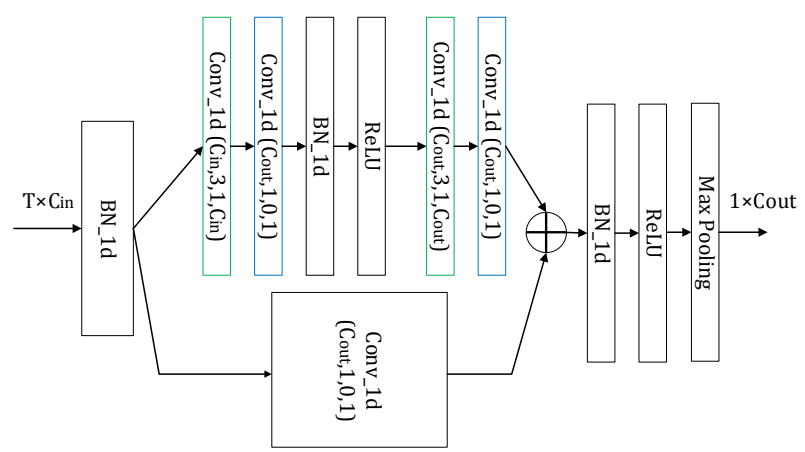

(a) Temporal Xception block configuration
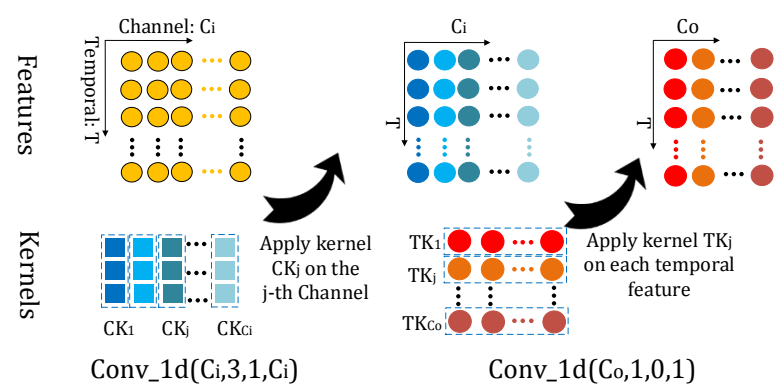

Conv_1d(Co,1,0,1)

(b) Channel- and temporal-wise convolution

Figure 3: Temporal Xception block (TXB). The detailed configuration of our proposed temporal Xception block is shown in (a). The parameters in the bracket denotes (\#kernel, kernel size, padding, \#groups) configuration of 1D convolution. Blocks in green denote channel-wise 1D convolutions and blocks in blue denote temporal-wise 1D convolutions. (b) depicts the channel-wise and temporal-wise 1D convolution. Input to TXB is feature sequence of a video, which is denoted as a $T \times C_{i n}$ tensor. Every kernel of channel-wise 1D convolution is applied along the temporal dimension within only one channel. Temporal-wise 1D convolution kernel convolves across all the channels along every temporal step.

so we set both spatial kernel size of a 3D convolution as 1 to save computation cost while the temporal kernel size is empirically set to be 3 . Applying 2 temporal convolutions on the $T$ local spatial-temporal feature maps after Res 3 and Res4 blocks introduces very limited extra computation cost but is effective to capture global spatial-temporal correlation progressively. In the temporal modeling blocks, weights of Conv3d layers are initially set to $1 /\left(3 \times C_{i}\right)$, where $C_{i}$ denotes input channel size, and biases are set to 0 . BN3d is initialized to be an identity mapping.

Temporal Xception Block: Our temporal Xception block is designed for efficient temporal modeling among feature sequence and easy optimization in an end-to-end manner. We choose temporal convolution to capture temporal relations instead of recurrent architectures mainly for the end-toend training purpose. Unlike ordinary 1D convolution which captures the channel-wise and temporal-wise information jointly, we decouple channel-wise and temporal-wise calculation for computational efficiency.

The temporal Xception architecture is shown in Fig.3(a). The feature sequence is viewed as a $T \times C_{i n}$ tensor, which is obtained by globally average pooling from the feature maps of $T$ super-images. Then, 1D batch normalization (Ioffe and Szegedy 2015) along the channel dimension is applied to such an input to handle the well-known co-variance shift issue, the output signal is $V$,

$$
v^{i}=\frac{u^{i}-m}{\sqrt{v a r}} * \alpha+\beta
$$

where $v^{i}$ and $u^{i}$ denote the $i^{t h}$ row of the output and input signals, respectively; $\alpha$ and $\beta$ are trainable parameters, $m$ and $v a r$ are accumulated running mean and variance of input mini-batches. To model temporal relation, convolutions along the temporal dimension are applied to $V$. We decouple temporal convolution into separate channel-wise and temporal-wise 1D convolutions. Technically, for channelwise 1D convolution, the temporal kernel size is set to 3, and the number of kernels and the group number are set to be the same with the input channel number. In this sense, every kernel convolves over temporal dimension within a single channel. For temporal-wise 1D convolution, we set both the kernel size and the group number to 1 , so that temporalwise convolution kernels operate across all elements along the channel dimension at each time step. Formally, channelwise and temporal-wise convolution can be described with Eq.2 and Eq.3, respectively,

$$
\begin{gathered}
y_{i, j}=\sum_{k=0}^{2} x_{k+i-1, j} * W_{j, k, 0}^{(c)}+b_{j}^{(c)}, \\
y_{i, j}=\sum_{k=0}^{C_{i}} x_{i, k} * W_{j, 0, k}^{(t)}+b_{j}^{(t)},
\end{gathered}
$$

where $x \in R^{T \times C_{i}}$ is the input $C_{i}$-Dim feature sequence of length $T, y \in R^{T \times C_{o}}$ denotes output feature sequence and $y_{i, j}$ is the value of the $j^{\text {th }}$ channel of the $i^{t h}$ feature, $*$ denotes multiplication. In Eq. $2, W^{(c)} \in R^{C_{o} \times 3 \times 1}$ denotes the channel-wise Conv kernel of (\#kernel, kernel size, \#groups $)=\left(C_{o}, 3, C_{i}\right)$. In Eq. $3, W^{(t)} \in R^{C_{o} \times 1 \times C_{i}}$ denotes the temporal-wise Conv kernel of (\#kernel, kernel size, \#groups $)=\left(C_{o}, 1,1\right) . b$ denotes bias. In this paper, $C_{o}$ is set to 1024. An intuitive illustration of separate channel- and temporal-wise convolution can be found in Fig.3(b).

As shown in Fig.3(a), similar to the bottleneck design of (He et al. 2016), the temporal Xception block has a long branch and a short branch. The short branch is a single 1D temporal-wise convolution whose kernel size and group size are both 1 . Therefore, the short branch has a temporal receptive field of 1 . Meanwhile, the long branch contains two channel-wise 1D convolution layers and thus has a temporal receptive filed of 5. The intuition is that, fusing branches with different temporal receptive field sizes is helpful for better temporal dynamics modeling. The output feature of 


\begin{tabular}{c|c|c}
\hline \hline Configuration & Top-1 & \# Params \\
\hline TSN (Backbone) & 73.02 & - \\
\hline w/o 1D BatchNorm & 73.55 & - \\
w/o C-Conv & 74.14 & - \\
w/o T-Conv & 74.06 & - \\
w/o Short-Branch & 74.33 & - \\
w/o Long-Branch & 74.21 & - \\
\hline Ordinary Temporal-Conv & 74.28 & $9.6 \mathrm{M}$ \\
LSTM & 73.21 & $10.9 \mathrm{M}$ \\
GRU & 73.66 & $8.3 \mathrm{M}$ \\
\hline proposed TXB & $\mathbf{7 4 . 6 2}$ & $\mathbf{4 . 6 M}$ \\
\hline
\end{tabular}

Table 1: Ablation study of TXN on Kinetics400. C-Conv and T-Conv denote channel-wise and temporal-wise 1D Conv, respectively.Prec@1 and number of model parameters are reported in the table.

the temporal Xception block is fed into a 1D max-pooling layer along the temporal dimension, and the pooled output is used as the spatial-temporal aggregated descriptor for classification.

\section{Experiments}

\subsection{Datasets and Evaluation Metric}

To evaluate the performance of our proposed StNet framework for large scale video-based action recognition, we perform extensive experiments on the recent large scale action recognition dataset named Kinetics (Kay et al. 2017). The first version of this dataset (denoted as Kinetics400) has 400 human action classes, with more than 400 clips for each class. The validation set of Kinetics400 consists of about $20 \mathrm{~K}$ video clips. The second version of Kinetics (denoted as Kinetics600) contains 600 action categories and there are about $400 \mathrm{~K}$ trimmed video clips in its training set and $30 \mathrm{~K}$ clips in the validation set. Due to unavailability of ground truth annotations for testing set, the results on the Kinetics dataset in this paper are evaluated on its validation set.

To validate that the effectiveness of StNet could be transferred to other datasets, we conduct transfer learning experiments on the UCF101 (Soomro, Zamir, and Shah 2012), which is much smaller than Kinetics. It contains 101 human action categories and 13,320 labeled video clips in total. The labeled video clips are divided into three training/testing splits for evaluation. In this paper, the evaluation metric for recognition effectiveness is average class accuracy, we also report total number of model parameters as well as FLOPs (total number of float-point multiplications executed in the inference phase) to depict model complexity.

\subsection{Ablation Study}

Temporal Xception Block: We conduct ablation experiments on our proposed temporal Xception block. To show the contribution of each component in TXB, we disable each of them one by one, and then train and test the models on the RGB feature sequence, which is extracted from the GlobalAvgPool layer of InceptionResnet-V2-TSN (Wang et

\begin{tabular}{c|c|c|c}
\hline \hline \multicolumn{3}{|c|}{ Configurations } & \multirow{2}{*}{ Top-1 } \\
\cline { 1 - 3 } Super-Image & TM Blocks & TXB & \\
\hline$\times(\mathrm{N}=1)$ & $\times$ & $\times$ & 72.2 \\
$\sqrt{ }(\mathrm{N}=5)$ & $\times$ & $\times$ & 74.2 \\
$\sqrt{ }(\mathrm{N}=5)$ & $\sqrt{ }$ & $\times$ & 76.0 \\
$\sqrt{ }(\mathrm{N}=5)$ & $\sqrt{ }$ & $\sqrt{ }$ & 76.3 \\
\hline
\end{tabular}

Table 2: Results evaluated on Kinetics600 validation set with different network configurations and $T=7$.

al. 2016) model trained on the Kinetics 400 dataset. Besides, we also implemented an ordinary 2-layered temporal Conv model (denoted as Ordinary Temporal-Conv) and RNN-based models (LSTM (Hochreiter and Schmidhuber 1997) and GRU (Cho et al. 2014)) for comparison. In Ordinary Temporal-Conv model, we replace the temporal Xception module by two 1D convolution layers, whose kernel size is 3 and output channel number is 1024 , to temporally model the input feature sequence. In this experiment, the hidden units of LSTM and GRU is set to 1024. The final classification results are predicted by a fully connected layer with output size of 400 . For each video, features of 25 frames are evenly sampled from the whole feature sequence to train and test all the models. The evaluation results and number of parameters of these models are reported in Table. 1

From the top lines of Table.1, we can see that each component contributes to the proposed TXB framework. Batch normalization handles the co-variance shift issue and it brings $1.07 \%$ absolute top- 1 accuracy improvement for RGB stream. Separate channel-wise and temporal-wise convolution layers is helpful for modeling temporal relations, and recognition performance drops without either of them. The results also demonstrate that our design of long-branch plus short-branch is useful by mixing multiple temporal receptive field encodings. Comparing the results listed in the middle lines with that of our TXB, it is clear that TXB achieves the best top- 1 accuracy among these models and the model is the smallest (with only 4.6 million parameters in total), especially, the gain over backbone TSN is up to 1.6 percent.

Study on Each Component in StNet: In this section, a series of ablation studies are performed to understand the importance of each design choice of our proposed StNet. To this end, we train multiple variants of our model to show how the performance is improved with our proposed super-image, temporal modeling blocks and temporal Xception block, respectively. There are chances that some tricks would be effective when either shallow backbone networks are used or evaluating on small datasets, therefore we choose to carry out experiments on the very large Kinetics600 dataset and the backbone we used is the very deep and powerful InceptionResnet-V2 (Szegedy et al. 2017). SuperImage (SI), temporal modeling blocks (TM), and temporal Xception block (TXB) are enabled one after another to generate 4 network variants and in this experiment, $T$ is set to 7 and $N$ to 5. The video frames are scaled such that their short-size is 331 and a random and the central $299 \times 299$ patch is cropped from each of the $T$ frames in the training phase and testing phase, respectively. 


\begin{tabular}{|c|c|c|c|c|c|c|}
\hline Framework & Backbone & Input $\times$ \# Clips & Dataset & Prec@1 & \# Params & FLOPs \\
\hline C2D (Wang et al. 2018b) & $\begin{array}{l}\text { ResNet50 } \\
\text { ResNet50 }\end{array}$ & $\begin{array}{c}{[32 \times 3 \times 256 \times 256] \times 1} \\
{[32 \times 3 \times 256 \times 256] \times 10}\end{array}$ & \multirow{10}{*}{ K400 } & $\begin{array}{r}62.42^{\times} \\
69.90\end{array}$ & $24.27 \mathrm{M}$ & $\begin{array}{c}26.29 G^{\vee} \\
262.9 \mathrm{G}\end{array}$ \\
\hline & ResNet50 & {$[32 \times 3 \times 256 \times 256] \times 1$} & & $64.65^{x}$ & & $164.84 \mathrm{G}$ \\
\hline C3D (Wang et al. 2018b) & ResNet50 & {$[32 \times 3 \times 256 \times 256] \times 10$} & & $71.86^{\sqrt{ }}$ & $35 \mathrm{MI}$ & $1648.4 G^{\times}$ \\
\hline I3D (Carreira and Zisserman) & BN-Inception & {$[$ All $\times 3 \times 256 \times 256] \times 1$} & & 70.24 & $12.7 M^{\checkmark}$ & $544.44 G^{\times}$ \\
\hline S3D(Xie et al.) & BN-Inception & {$[$ All $\times 3 \times 224 \times 224] \times 1$} & & $72.20^{\checkmark}$ & $8.8 M^{\checkmark}$ & $518.6 G^{\times}$ \\
\hline MF-Net(Chen et al.) & & $\begin{array}{c}{[16 \times 3 \times 224 \times 224] \times 1} \\
{[16 \times 3 \times 224 \times 224] \times 50}\end{array}$ & & $\begin{array}{l}65.00^{\times} \\
72.80^{\sqrt{ }}\end{array}$ & $8.0 M^{\checkmark}$ & $\begin{array}{l}11.1 G^{\vee} \\
555 G^{\times}\end{array}$ \\
\hline R(2+1)D-RGB (Tran et al.) & ResNet34 & {$[32 \times 3 \times 112 \times 112] \times 10$} & & $72.00 \sqrt{ }$ & $63.8 \mathrm{M}$ & $1524 G^{\times}$ \\
\hline ARTNet-RGB (Wang et al.) & ResNet18 & {$[16 \times 3 \times 112 \times 112] \times 25$} & & $70.70^{\checkmark}$ & $35.2 \mathrm{M}$ & $593 G^{\times}$ \\
\hline Nonlocal-I3D (Wang et al.) & ResNet50 & $\begin{array}{c}{[128 \times 3 \times 224 \times 224] \times 1} \\
{[128 \times 3 \times 224 \times 224] \times 30}\end{array}$ & & $\begin{array}{l}67.30^{\times} \\
76.50^{\vee}\end{array}$ & $35.33 \mathrm{M}$ & $\begin{array}{c}145.7 \mathrm{G} \\
4371 G^{\times}\end{array}$ \\
\hline StNet (Ours) & $\begin{array}{l}\text { ResNet50 } \\
\text { ResNet101 }\end{array}$ & $\begin{array}{l}{[25 \times 15 \times 256 \times 256] \times 1} \\
{[25 \times 15 \times 256 \times 256] \times 1}\end{array}$ & & $\begin{array}{l}69.85 \\
71.38\end{array}$ & & $\begin{array}{l}\text { 189.29G } \\
\text { 310.50G }\end{array}$ \\
\hline TSN (Wang et al. 2016) & $\begin{array}{c}\text { IRv2 } \\
\text { SE-ResNeXt152 }\end{array}$ & $\begin{array}{l}{[25 \times 3 \times 331 \times 331] \times 1} \\
{[25 \times 3 \times 256 \times 256] \times 1}\end{array}$ & \multirow{4}{*}{ K600 } & $\begin{array}{l}76.22 \\
76.16 \\
\end{array}$ & $\begin{array}{c}55.23 \mathrm{M} \\
142.94 M^{\times}\end{array}$ & $\begin{array}{c}410.85 \mathrm{G} \\
875.21 G^{\times}\end{array}$ \\
\hline I3D (Carreira et al.) & BN-Inception & {$[$ All $\times 3 \times 256 \times 256] \times 1$} & & $71.9^{\times}$ & $12.90 M^{\sqrt{ }}$ & $544.45 \mathrm{G}$ \\
\hline P3D (Yao and Li 2018) & $\begin{array}{c}\text { ResNet152 } \\
\text { U }\end{array}$ & $\begin{array}{c}{[32 \times 3 \times 299 \times 299] \times 1} \\
{[128 \times 3 \times \mathrm{U} \times \mathrm{U}] \times \mathrm{U}}\end{array}$ & & $\begin{array}{l}71.31^{\times} \\
77.94^{\vee}\end{array}$ & $\begin{array}{c}66.90 \mathrm{M} \\
\mathrm{U}\end{array}$ & $132.38 G^{\vee}$ \\
\hline StNet (Ours) & $\begin{array}{l}\text { SE-ResNeXt101 } \\
\text { IRv2 }\end{array}$ & $\begin{array}{l}{[25 \times 15 \times 256 \times 256] \times 1} \\
{[25 \times 15 \times 331 \times 331] \times 1}\end{array}$ & & $\begin{array}{c}76.04 \\
\mathbf{7 8 . 9 9}\end{array}$ & $\begin{array}{l}79.13 \mathrm{M} \\
\mathbf{7 2 . 1 3 M}\end{array}$ & $\begin{array}{l}453.95 \mathrm{G} \\
\mathbf{4 3 9 . 5 7 G}\end{array}$ \\
\hline
\end{tabular}

Table 3: Comparison of StNet and several state-of-the-art 2D/3D convolution based solutions. The results are reported on validation set of Kinetics400 and Kinetics600, with RGB modality only. We investigate both Prec@1 and model efficiency w.r.t. total number of model parameters and FLOPs in inference. Here, "IRv2" denotes InceptionResNet-V2, "K400" is short for Kinetics400 and so is the case for "K600". U denotes unknown. "All" means using all frames in a video.

Experiment results are reported in Table.2. When the three components are all disabled, the model degrades to be TSN (Wang et al. 2016), which achieves top-1 precision of $72.2 \%$ on Kinetics600 validation set. By enabling super-image, the recognition performance is improved by $2.0 \%$ and the gain comes from introducing local spatial-temporal modelling. When the two temporal modelling blocks are inserted, the Prec@1 is further boosted to $76.0 \%$, it evidences the fact that modeling global spatial-temporal interactions among the feature maps of super-images is necessary for performance improvement, because it can well represent highlevel video features. The final performance is $76.3 \%$ when all the components are integrated and this shows that using TXB to capture long-term temporal dynamics is still a plus even if local and global spatial-temporal relationship is modeled by enabling super-images and temporal modeling blocks.

\subsection{Comparison with Other Methods}

We evaluate the proposed framework against the recent state-of-the-art 2D/3D convolution based solutions. Extensive experiments are conducted on the datasets of Kinetics400 and Kinetics600 to make comparisons among these models in terms of their effectiveness (i.e., top-1 accuracy) and efficiency (reflected by total number of model parameters and FLOPs needed in the inference phase). To make thorough comparison, we evaluated different methods with several relatively small backbone networks and a few very deep backbones on Kinetics400 and Kinetics600, respectively. Results are summarized in Table.3. Numbers with $\checkmark$ mean that the results are pleasing and numbers with ${ }^{\times}$represent unsatisfying ones. From the evaluation results, we can draw the following conclusions:

StNet outperforms 2D-Conv based solution: (1) C2DResNet50 is cheap in FLOPs, but its top-1 recognition precision is very poor $(62.42 \%)$ if only 1 clip is tested. When 10 clips are tested, the performance is boosted to $69.9 \%$ at the cost of 262.9G FLOPs. StNet-ResNet50 achieves Prec@1 of $69.85 \%$ and only 189.29 G FLOPs are needed. (2) When large backbone models are used, StNet still outperforms 2DConv based solution, this can be concluded from the fact that StNet-IRv2 significantly boosts the performance of TSNIRv2 (which is $76.22 \%$ ) to $78.99 \%$ while the total number of FLOPs is slightly increased from $410.85 \mathrm{G}$ to $439.57 \mathrm{G}$. Besides, StNet-SE-ResNeXt101 performs comparable with TSN-SE-ResNeXt152, but the model size and FLOPs are significantly saved (from $875.21 \mathrm{G}$ to $453.95 \mathrm{G}$ ).

StNet obtains a better trade off than 3D-Conv: (1) Though model size and recognition performance of I3D is very plausible, it is very costly. C3D-ResNet50 achieves Prec@1 of $64.65 \%$ with single clip test. With comparable FLOPs, StNet-ResNet50 achieves $69.85 \%$ which is much better than C3D-ResNet50. Ten clip test significantly improves performance to $71.86 \%$, however, the FLOPs is as huge as 1648.4G. StNet-ResNet101 achieves $71.38 \%$ with over 5x FLOPs reduction. (2) Compared with P3D- 


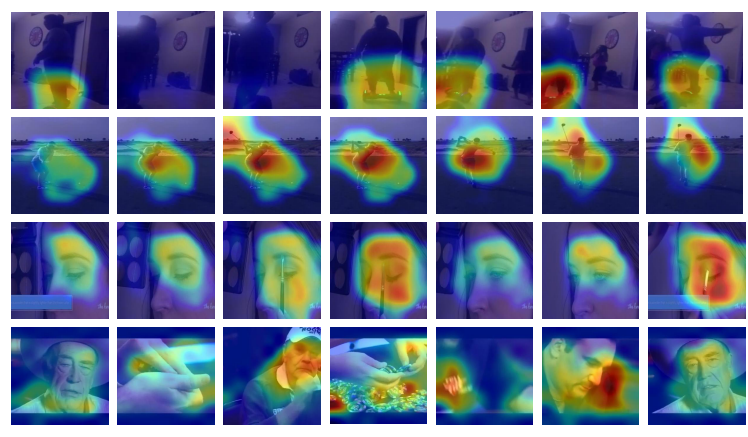

(a) Activation maps of TSN
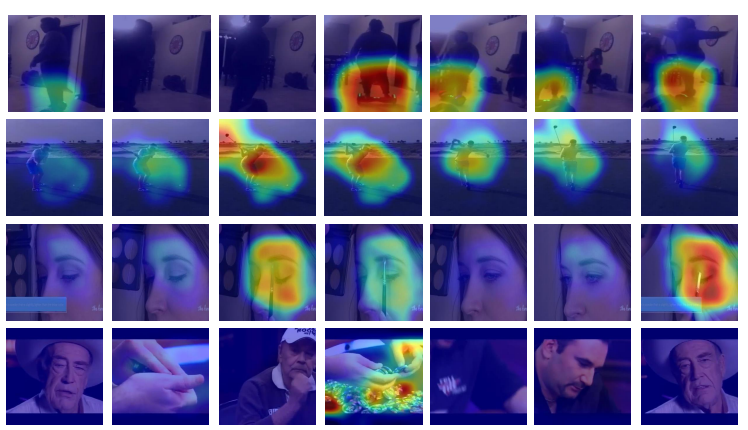

(b) Activation maps of StNet

Figure 4: Visualizing action-specific activation maps with the CAM (Zhou et al. 2016). Four action classes, i.e., hoverboarding, golf driving ,filling eyebrows and playing poker, are shown from top to the bottom. It is clear that StNet can well capture temporal dynamics in video and focuses on the spatial-temporal regions which really corresponds to the action class.

ResNet152, StNet-IRv2 outperforms by a large margin (78.99\% v.s. 71.31) with acceptable FLOPs increase (from 132.38G to $439.57 \mathrm{G})$. Besides, Single clip test performance of StNet-IRv2 still outperforms P3D by $1.05 \%$, which is used for the Kinetics600 challenge with 128 frames input and no further details about its backbone, input size as well as number of testing clips. (3) Compared with S3D (Xie et al. 2018), R(2+1)D(Tran et al. 2018), MF-Net (Chen et al. 2018) and Nonlocal-I3d (Wang et al. 2018b), the proposed StNet can still strike good performance-FLOPs trade-off.

\subsection{Transfer Learning on UCF101}

We transfer RGB models of StNet pre-trained on Kinetics to the much smaller dataset of UCF101 (Soomro, Zamir, and Shah 2012) to show that the learned representation can be well generalized to other dataset. The results included in Table 4 are the mean class accuracy from three training/testing splits. It is clear that our Kinetics pre-trained StNet models with ResNet50, ResNet101 and the large InceptionResNetV2 backbone demonstrate very powerful transfer learning capability, and mean class accuracy is up to $93.5 \%, 94.3 \%$ and $95.7 \%$, respectively. Specifically, the transferred StNetIRv2 RGB model achieves the state-of-the-art performance while its FLOPs is $123 \mathrm{G}$.

\begin{tabular}{c|c|c|c}
\hline \hline Model & Pre-Train & FLOPs & Accuracy \\
\hline C3D+Res18 & K400 & - & 89.8 \\
\hline I3D+BNInception & K400 & $544 G^{\times}$ & 95.6 \\
\hline TSN+BNInception & K400 & - & 91.1 \\
TSN+IRv2 (T=25) & K400 & $411 \mathrm{G}$ & 92.7 \\
\hline StNet-Res50 (T=7) & K400 & $53 G^{\checkmark}$ & $\mathbf{9 3 . 5}$ \\
StNet+Res101 (T=7) & K400 & $\mathbf{8 7 G}$ & $\mathbf{9 4 . 3}$ \\
StNet+IRv2 (T=7) & K600 & $\mathbf{1 2 3 G}$ & $\mathbf{9 5 . 7}$ \\
\hline \hline
\end{tabular}

Table 4: Mean class accuracy of different models transferred on UCF101. RGB frames of UCF101 are used for training and testing. The mean class accuracy averaged over the three splits of UCF101 is reported.

\subsection{Visualization in StNet}

To help us better understand how StNet learns discriminative spatial-temporal descriptors for action recognition, we visualize the class-specific activation maps of our model with the CAM (Zhou et al. 2016) approach. In this experiment, we set $T$ to 7 and the 7 snippets are evenly sampled from video sequences in the Kinetics600 validation set to obtain their class-specific activation map. As a comparison, we also visualize the activation maps of TSN model, which exploits local spatial information and then fuses $T$ snippets by averaging classification score rather than jointly modeling local and global spatial-temporal information. As an illustration, Fig. 4 lists activation maps of four action classes (hoverboarding, golf driving, filling eyebrows and playing poker) of both models.

These maps shows that, compared to TSN which fails to jointly model local and global spatial-temporal dynamics in videos, our StNet can capture the temporal interactions inside video frames. It focuses on the spatial-temporal regions which are closely related to the groundtruth action. For example, it pays more attention to faces with eyebrow pencil in the nearby while regions with only faces are not so activated. Particularly, in the "play poker" example, StNet is significantly activated only by the hands and casino tokens. Nevertheless, TSN is activated by many regions of faces.

\section{Conclusion}

In this paper, we have proposed the StNet for joint local and global spatial-temporal modeling to tackle the problem of action recognition in videos. Local spatial-temporal information is modeled by applying $2 \mathrm{D}$ convolutions on sampled super-images, while global temporal interactions are encoded by temporal convolutions on the local spatialtemporal feature maps of super-images. Besides, we propose a temporal Xception block to enhance the capacity of modeling temporal dynamics. Such design choices make our model relatively lightweight and computationally efficient in both training and inference phases. So it allows for instantiating StNet with the most powerful 2D CNNs in large 
scale dataset and boosting the final action recognition accuracy. Extensive experiments on large scale action recognition benchmark Kinetics have verified the effectiveness of StNet. In addition, StNet trained on Kinetics exhibits pretty good transfer learning ability on the UCF101 dataset.

\section{References}

Arandjelovic, R.; Gronat, P.; Torii, A.; Pajdla, T.; and Sivic, J. 2016. Netvlad: Cnn architecture for weakly supervised place recognition. In CVPR, 5297-5307.

Carreira, J., and Zisserman, A. 2017. Quo vadis, action recognition? a new model and the kinetics dataset. In CVPR, 4724-4733.

Carreira, J.; Noland, E.; Banki-Horvath, A.; Hillier, C.; and Zisserman, A. 2018. A short note about kinetics-600. arXiv preprint arXiv:1808.01340.

Chen, Y.; Kalantidis, Y.; Li, J.; Yan, S.; and Feng, J. 2018. Multifiber networks for video recognition. In ECCV, 352-367.

Cho, K.; Van Merriënboer, B.; Bahdanau, D.; and Bengio, Y. 2014. On the properties of neural machine translation: Encoder-decoder approaches. arXiv preprint arXiv:1409.1259.

Chollet, F. 2017. Xception: Deep learning with depthwise separable convolutions. In CVPR, 1800-1807.

Dalal, N.; Triggs, B.; and Schmid, C. 2006. Human detection using oriented histograms of flow and appearance. In ECCV, 428-441. Springer.

Diba, A.; Sharma, V.; and Van Gool, L. 2017. Deep temporal linear encoding networks. In CVPR, 1541-1550.

Donahue, J.; Anne Hendricks, L.; Guadarrama, S.; Rohrbach, M.; Venugopalan, S.; Saenko, K.; and Darrell, T. 2015. Long-term recurrent convolutional networks for visual recognition and description. In $C V P R, 2625-2634$.

Feichtenhofer, C.; Pinz, A.; and Wildes, R. 2016. Spatiotemporal residual networks for video action recognition. In NIPS, 34683476.

Feichtenhofer, C.; Pinz, A.; and Wildes, R. P. 2017. Temporal residual networks for dynamic scene recognition. In $C V P R, 4728-$ 4737.

Fernando, B.; Gavves, E.; Oramas, J. M.; Ghodrati, A.; and Tuytelaars, T. 2015. Modeling video evolution for action recognition. In CVPR, 5378-5387.

Girdhar, R.; Ramanan, D.; Gupta, A.; Sivic, J.; and Russell, B. 2017. Actionvlad: Learning spatio-temporal aggregation for action classification. In CVPR, 3165-3174.

He, K.; Zhang, X.; Ren, S.; and Sun, J. 2016. Deep residual learning for image recognition. In CVPR, 770-778.

Hochreiter, S., and Schmidhuber, J. 1997. Long short-term memory. Neural computation 9(8):1735-1780.

Hu, J.; Shen, L.; and Sun, G. 2018. Squeeze-and-excitation networks. In $C V P R, 7132-7141$.

Ioffe, S., and Szegedy, C. 2015. Batch normalization: Accelerating deep network training by reducing internal covariate shift. In ICML, 448-456.

Karpathy, A.; Toderici, G.; Shetty, S.; Leung, T.; Sukthankar, R.; and Fei-Fei, L. 2014. Large-scale video classification with convolutional neural networks. In CVPR, 1725-1732.

Kay, W.; Carreira, J.; Simonyan, K.; Zhang, B.; Hillier, C.; Vijayanarasimhan, S.; Viola, F.; Green, T.; Back, T.; Natsev, P.; et al. 2017. The kinetics human action video dataset. arXiv preprint arXiv:1705.06950.
Klaser, A.; Marszałek, M.; and Schmid, C. 2008. A spatiotemporal descriptor based on 3d-gradients. In $B M V C, 1-10$.

Long, X.; Gan, C.; de Melo, G.; Wu, J.; Liu, X.; and Wen, S. 2018. Attention clusters: Purely attention based local feature integration for video classification. In CVPR, 7834-7843.

Qiu, Z.; Yao, T.; and Mei, T. 2017. Learning spatio-temporal representation with pseudo-3d residual networks. In ICCV, 5534-5542. IEEE.

Scovanner, P.; Ali, S.; and Shah, M. 2007. A 3-dimensional sift descriptor and its application to action recognition. In $A C M M M$, 357-360.

Shi, Y.; Tian, Y.; Wang, Y.; Zeng, W.; and Huang, T. 2017. Learning long-term dependencies for action recognition with a biologically-inspired deep network. In ICCV, 716-725.

Simonyan, K., and Zisserman, A. 2014. Two-stream convolutional networks for action recognition in videos. In NIPS, 568-576.

Soomro, K.; Zamir, A. R.; and Shah, M. 2012. Ucf101: A dataset of 101 human actions classes from videos in the wild. arXiv preprint arXiv:1212.0402.

Szegedy, C.; Ioffe, S.; Vanhoucke, V.; and Alemi, A. A. 2017. Inception-v4, inception-resnet and the impact of residual connections on learning. In $A A A I, 4278-4284$.

Tran, D.; Bourdev, L.; Fergus, R.; Torresani, L.; and Paluri, M. 2015. Learning spatiotemporal features with $3 d$ convolutional networks. In ICCV, 4489-4497.

Tran, D.; Wang, H.; Torresani, L.; Ray, J.; LeCun, Y.; and Paluri, M. 2018. A closer look at spatiotemporal convolutions for action recognition. In $C V P R, 6450-6459$.

Wang, H., and Schmid, C. 2013. Action recognition with improved trajectories. In ICCV, 3551-3558.

Wang, L.; Xiong, Y.; Wang, Z.; Qiao, Y.; Lin, D.; Tang, X.; and Van Gool, L. 2016. Temporal segment networks: Towards good practices for deep action recognition. In ECCV, 20-36.

Wang, L.; Li, W.; Li, W.; and Van Gool, L. 2018a. Appearance-andrelation networks for video classification. In CVPR, 1430-1439.

Wang, X.; Girshick, R.; Gupta, A.; and He, K. 2018b. Non-local neural networks. In CVPR, 7794-7803.

Wang, L.; Qiao, Y.; and Tang, X. 2016. MoFAP: A multi-level representation for action recognition. International Journal of Computer Vision 119(3):254-271.

Xie, S.; Girshick, R.; Dollár, P.; Tu, Z.; and He, K. 2017. Aggregated residual transformations for deep neural networks. In $C V P R$, 5987-5995.

Xie, S.; Sun, C.; Huang, J.; Tu, Z.; and Murphy, K. 2018. Rethinking spatiotemporal feature learning: Speed-accuracy trade-offs in video classification. In ECCV, 305-321.

Yao, T., and Li, X. 2018. Yh technologies at activitynet challenge 2018. arXiv preprint arXiv:1807.00686.

Yue-Hei Ng, J.; Hausknecht, M.; Vijayanarasimhan, S.; Vinyals, O.; Monga, R.; and Toderici, G. 2015. Beyond short snippets: Deep networks for video classification. In CVPR, 4694-4702.

Zhang, B.; Wang, L.; Wang, Z.; Qiao, Y.; and Wang, H. 2016. Real-time action recognition with enhanced motion vector CNNs. In CVPR, 2718-2726.

Zhou, B.; Khosla, A.; Lapedriza, A.; Oliva, A.; and Torralba, A. 2016. Learning deep features for discriminative localization. In CVPR, 2921-2929.

Zolfaghari, M.; Singh, K.; and Brox, T. 2018. Eco: Efficient convolutional network for online video understanding. In ECCV. 